\title{
Causally simple inextendible spacetimes are hole-free
}

\author{
E. Minguzzi* \\ Dipartimento di Matematica Applicata "G. Sansone", \\ Università degli Studi di Firenze, Via S. Marta 3, I-50139 Firenze, Italy.
}

It is shown that causally simple inextendible spacetimes are hole-free, thus confirming the expectation that causal simplicity removes holes from spacetime. This result is optimal in the sense that causal simplicity cannot be weakened to causal continuity. Physically, it means that if there is some partial Cauchy hypersurface which, for some reason, does not fully develop its influence, then there is some discontinuity in the causal relation.

\section{INTRODUCTION}

With the general theory of relativity we learned to represent events as points of a connected Hausdorff time oriented Lorentzian manifold. We call these manifolds spacetimes. However, it is easy to recognize that this category of manifolds is too large. Indeed, removing points from one spacetime leaves us with another spacetime, thus, taking the mathematical model seriously, one should conclude that spacetime might suddenly end without giving any warning to the observers living in it. It seems that to accept this type of drastic behavior would mean to dismiss the possibility of making predictions. Therefore, there is a certain agreement that the above notion of spacetime should be narrowed with the imposition of additional conditions. One such condition is inextendibility, namely, the Lorentzian manifold should not be a subset of a larger one. Physically, this condition assures that spacetime will not end for some observer (i.e. timelike worldline) if there is the possibility of hosting the observer by enlarging the manifold.

Another, less known, but soundly motivated condition is that of hole-freeness. An inextendible spacetime is hole-free if the domain of dependence of any partial Cauchy hypersurface cannot be extended by imbedding it into a different spacetime. In essence it states that any Cauchy development on spacetime must be maximal (in the sense of partial orders) among the Cauchy developments of that hypersurface (in possibly different spacetimes). Philosophically speaking, it means that spacetime evolves as much as possible, without 'forgetting' any event that it can possibly determine. This idea was introduced by Geroch in order to identify some pathological spacetimes which could not be physically dismissed on other grounds. According to Geroch, general relativity must be complemented with the condition of hole-freeness.

The inextendibility condition allows us to discard spacetimes which are proper subsets of physically reasonable spacetimes. The hole-free condition has a similar effect but can be formulated independently of the inextendibility condition (see the examples of section II A). Since both should be imposed by fiat, they should most often appear in conjunction.

Causal simplicity is another condition which, intuitively, forbids the removal of points from spacetime. Indeed, the levels of the causal ladder [1, 2] above stable causality, such

*ettore.minguzzi@unifi.it 
as causal continuity, causal simplicity and global hyperbolicity appear as increasingly refined conditions which decrease the influence of points at infinity on spacetime, and hence, in particular, of those points at infinity obtained by removing interior spacetime points. Causal simplicity seems to work perfectly fine on known examples although, contrary to hole-freeness, it cannot be imposed by fiat and could fail even in physically meaningful spacetimes (e.g. plane-fronted waves).

In this work I wish to show that, as examples suggest, causal simplicity together with inextendibility implies hole-freeness. I will also comment on previous variants on the definition of hole-free spacetime.

I denote with $(M, g)$ a spacetime (connected, Hausdorff, time-oriented Lorentzian manifold without boundary), of arbitrary dimension $n \geq 2$ having a metric $g$ of signature $(-,+, \ldots,+)$. Unless otherwise specified, all the causal curves that we shall consider are future directed, thus a past inextendible curve ends at its future endpoint. Sometimes by geodesic we shall actually mean pregeodesic. The subset symbol $\subset$ is reflexive, $X \subset X$.

\section{HOLE-FREE SPACETIMES}

The future domain of dependence (or future Cauchy development) $D^{+}(S)$ of a set $S$ is made by all the points $p \in M$ for which any inextendible $\left(C^{1}\right)$ causal curve through $p$ intersects $S$. The set $\tilde{D}^{+}(S)$ is defined analogously, where causal is replaced by timelike. [1] If $S$ is topologically closed as a subset of $M$ then $\overline{D^{+}(S)}=\tilde{D}^{+}(S)$. A dual definition of past domain of dependence $D^{-}(S)$ can be given. The (total) domain of dependence is $D(S)=D^{+}(S) \cup D^{-}(S)$.

An acausal edgeless (and hence topologically closed) set is a partial Cauchy hypersurface. For the definition of edge of an achronal set see[1]. Essentially, the edge is the boundary of the achronal set $S$ with respect the the topology induced on an maximal achronal set $A$ containing $S$.

Let $S$ be acausal. It is well known that through each point of $H^{+}(S)$ passes a past inextendible lightlike geodesic generator whose intersection with $\bar{S}$, whenever it exists, belongs to edge $(S)$ (and dually in the past case).

As a consequence, if $S$ is acausal and does not contain its edge (e.g. if $S$ is a partial Cauchy hypersurface) then $D(S)$ is open and non-empty (as it contains $S$ ), and furthermore $D(S) \cap H(S)=\emptyset$. We remark that we cannot weaken the acausality of $S$ to achronality if we want to assure that $D(S)$ is open.

A spacetime is globally hyperbolic iff we can find a partial Cauchy hypersurface such that $D(S)=M$. It is causally simple if it is causal and the sets $J^{ \pm}(x)$ are closed for every $x \in M$ (or equivalently, the causal relation $J^{+} \subset M \times M$ is closed [2]). It is causally continuous if it is weakly distinguishing and reflective. [3] Global hyperbolicity implies causal simplicity which implies casual continuity.

Any isometry is injective but not necessarily surjective. A spacetime $(M, g)$ is inextendible if it is not contained in a larger spacetime $\left(M^{\prime}, g^{\prime}\right)$, namely if we cannot find an isometry $\psi: M \rightarrow M^{\prime}$ such that $\psi(M) \neq M^{\prime}$.

Definition II.1. A spacetime $(M, g)$ has a future Cauchy hole (or simply a future hole) if there is a partial Cauchy hypersurface $S$ and an isometry $\varphi: \tilde{D}(S) \rightarrow N$, on a spacetime $(N, \sigma)$, such that $\varphi(S)$ is acausal and $\varphi\left(H^{+}(S)\right) \cap D^{+}(\varphi(S)) \neq \emptyset$. The definition of past 
Cauchy hole is given dually. A spacetime is Cauchy holed if it has a future or a past hole. A spacetime is (future/past) hole-free if it has no (future/past) hole.

Intuitively, $S$ generates a future hole if its Cauchy horizon $H^{+}(S)$, while outside the domain of influence of $S$ in $M$, enters into the domain of influence in an alternative spacetime, that is, prediction can be extended passing through the former horizon.

According to this definition the region $t<0$ of Minkowski spacetime is not holed (compare with previous definitions, see remark [I.7).

Proposition II.2. Let $S$ be a closed achronal set and let $\varphi: \tilde{D}(S) \rightarrow N$ be an isometry, then $\varphi(D(S)) \subset D(\varphi(S))$ and $\varphi(\tilde{D}(S)) \subset \tilde{D}(\varphi(S))$.

Proof. Let $q^{\prime} \in \varphi\left(D^{+}(S)\right) \backslash \varphi(S), q^{\prime}=\varphi(q), q \in D^{+}(S) \backslash S$. There is a neighborhood of $0 \in T M_{q}$, such that the exponential map of the past non-spacelike vectors in that neighborhood is contained in $D^{+}(S)$. By the isometry, the same is true for $q^{\prime}$ with respect to $\varphi\left(D^{+}(S)\right)$, in particular there is a neighborhood $U_{q^{\prime}}$ of $q^{\prime}$ such that $U_{q^{\prime}} \cap J^{-}\left(q^{\prime}\right) \subset \varphi\left(D^{+}(S)\right)$. Suppose by contradiction that $q^{\prime} \notin D^{+}(\varphi(S))$, then there is a past inextendible causal curve $\gamma^{\prime}:(0,1] \rightarrow N$ ending at $q^{\prime}$ and not intersecting $\varphi(S)$. A last segment of $\gamma^{\prime}$ must be contained in $\varphi\left(D^{+}(S)\right)$. The curve $\gamma:=\varphi^{-1} \circ \gamma^{\prime}$ is causal and ends at $q$ thus there is a segment of $\gamma$ which is ending at $q$ and entirely contained in $D^{+}(S)$. In fact there is a maximal segment of this type which provides a past inextendible causal curve ending at $q$ not intersecting $S$, a contradiction. The proof in the past and timelike cases are analogous.

If there is a hole the inclusion is strict.

Proposition II.3. Suppose that $(M, g)$ is (future/past) holed and let $\varphi$ be as in definition II.1, then $\varphi(D(S)) \subsetneq D(\varphi(S))$ (resp. $\varphi\left(D^{ \pm}(S)\right) \subsetneq D^{ \pm}(\varphi(S))$ ).

Proof. Indeed, by definition of hole $D(\varphi(S))$ contains some points of $\varphi(H(S))$ while $\varphi(D(S))$ does not contain any of them since $\varphi: \overline{D(S)} \rightarrow N$ is injective.

Remark II.4. Let $\varphi: \tilde{D}(S) \rightarrow N$ be an isometry with $S$ partial Cauchy hypersurface, and $\varphi(S)$ acausal set. Since $\varphi$ is an isometry, it is a local diffeomorphism and hence it is open. The set $D(S)$ is open thus $\varphi(D(S))$ is open. In particular, as $S \subset D(S), \varphi(S)$ is contained in the open set $\varphi(D(S))$ and no point of $\varphi(S)$ can belong to edge $(\varphi(S)$ ) (we recall [1, Prop. 6.5.2] that this set coincides with edge $(H(S))$ ). By hypothesis the hypersurface $\varphi(S)$ is acausal and furthermore, it does not contain its edge, thus $D(\varphi(S))$ is open and $H(\varphi(S)) \cap D(\varphi(S))=\emptyset$.

Proposition II.5. Suppose that $(M, g)$ is inextendible, $S$ is a partial Cauchy hypersurface and let $\varphi: \tilde{D}(S) \rightarrow N$ be an isometry on a spacetime $(N, \sigma)$, such that $\varphi(S)$ is acausal. Then

$$
\left[D^{+}(\varphi(S)) \cap \partial \varphi\left(D^{+}(S)\right)\right] \backslash \varphi(S)=D^{+}(\varphi(S)) \cap \overline{\varphi\left(H^{+}(S)\right)},
$$

and if $M$ is future holed then $S,(N, \sigma)$ and $\varphi$ can be chosen in such a way that this set is non-empty.

Proof. The definition of future hole implies that the right-hand side is non-empty for some choice of $S,(N, \sigma)$ and $\varphi$.

The set $\varphi\left(H^{+}(S)\right)$ has empty intersection with $\varphi\left(D^{+}(S)\right)$ because $\varphi: \overline{D^{+}(S)} \rightarrow N$ is injective. However, by continuity, since $H^{+}(S) \subset \overline{D^{+}(S)}$ we have $\varphi\left(H^{+}(S)\right) \subset \overline{\varphi\left(D^{+}(S)\right)}$, 
thus $\varphi\left(H^{+}(S)\right) \subset \overline{\varphi\left(D^{+}(S)\right)} \backslash \varphi\left(D^{+}(S)\right) \subset \partial \varphi\left(D^{+}(S)\right)$. Using the closure of the last set we get $\overline{\varphi\left(H^{+}(S)\right)} \subset \partial \varphi\left(D^{+}(S)\right)$ which proves $\supset$.

For the inclusion $\subset$, suppose that $\left.r \in D^{+}(\varphi(S)) \cap \partial \varphi\left(D^{+}(S)\right)\right] \backslash \varphi(S)$ and that $r \notin$ $\overline{\varphi\left(H^{+}(S)\right)}$. We can find an open neighborhood $U \ni r, U \subset I^{+}(\varphi(S)) \cap D^{+}(\varphi(S))$, such that $U \cap \varphi\left(H^{+}(S)\right)=\emptyset$. Gluing $U$ with $M$ by identifying $\varphi^{-1}(U) \subset D(S) \subset M$ with $\varphi\left(\varphi^{-1}(U)\right)$ through $\varphi$ we get an extension of $M$, a contradiction.

Proposition II.6. An inextendible spacetime $(M, g)$ is hole-free if and only if for every partial Cauchy hypersurface $S$, and isometry $\varphi: \tilde{D}(S) \rightarrow N$, on a spacetime $(N, \sigma)$, such that $\varphi(S)$ is acausal, we have $\varphi(D(S))=D(\varphi(S))$.

Proof. $\Leftarrow$. This direction is an easy consequence of Prop. II.3.

$\Rightarrow$. If $(M, g)$ has no hole then for every partial Cauchy hypersurface $S$ and isometry $\varphi: \tilde{D}(S) \rightarrow N$, on a spacetime $(N, \sigma)$, such that $\varphi(S)$ is acausal we have $\varphi\left(H^{ \pm}(S)\right) \cap$ $D^{ \pm}(\varphi(S))=\emptyset$. By Prop. 【I.5 we have $D(\varphi(S)) \cap \partial \varphi(D(S))=\emptyset$. Let us show that it implies $D(\varphi(S)) \subset \varphi(D(S))$. Indeed, suppose by contradiction that there is $q^{\prime} \in D(\varphi(S)) \backslash \varphi(D(S))$. We shall assume $q^{\prime} \in D^{+}(\varphi(S))$, the case $q^{\prime} \in D^{-}(\varphi(S))$ being analogous. There is a future directed timelike curve $\gamma^{\prime}:[0,1] \rightarrow D(\varphi(S))$ which starts from some point $p^{\prime} \in \varphi(S)$ and reaches $q^{\prime}$. Thus $\gamma^{\prime}$ starts from the open set $\varphi(D(S))$ and reaches a point $q^{\prime}$ outside it. Thus there is a first point $r=\gamma(b) \in D^{+}(\varphi(S)) \backslash \varphi(D(S)), b \in(0,1]$, hence belonging to $\left[\partial \varphi\left(D^{+}(S)\right)\right] \backslash \varphi(S)$. Observe that $r \in D^{+}(\varphi(S))$ since it belongs to $J^{-}\left(q^{\prime}\right) \cap I^{+}(\varphi(S))$. This fact contradicts $D(\varphi(S)) \cap \partial \varphi(D(S))=\emptyset$ and proves the inclusion $D(\varphi(S)) \subset \varphi(D(S))$. Using Prop. II.2 we obtain the equality $\varphi(D(S))=D(\varphi(S))$.

Remark II.7 (On definitions). A first definition of hole-free spacetime can be found in Geroch, [4] who should be credited with the introduction of the concept. He gives a definition similar to the characterizing property of Prop. II.6. In his definition there is no edgeless condition on $S$ and, furthermore, he uses $\tilde{D}(S)$ in place of $D(S)$ in the last equality, which causes some undesired consequences related to the non openness of $\tilde{D}(S)$ (see Manchak [5]). Manchak mentions a possible correction recently suggested by Geroch himself, which makes the definition almost coincident with the property of Prop. II.6 but for the fact that: (i) he uses the achronal condition on $S$, and expresses it using $\tilde{D}(S)$ and, (ii) he misses the inextendibility condition with the unfortunate consequence that the region $t<0$ of Minkowski spacetime becomes holed according to his definition, (iii) $\varphi$ is defined over $D(S)$ instead that over $\overline{D(S)}$. We choose to define $\varphi$ over $\overline{D(S)}$ because this choice allows us to skip some technical issues connected with the extension of the isometry from the open set $D(S)$ to its closure (by the way, Krasnikov requires the isometry to be defined in a neighborhood of $\overline{D(S)})$.

Another definition can be found in Clarke [6] where he credits J. Earman and N. Woodhouse (Earman refers to the hole-free condition as the determinism maximal property [7, Sect. 3.8]). However, Clarke uses spacelike hypersurfaces (without edge) in place of partial Cauchy hypersurfaces. Manchak points out that Clarke's definition runs into the same problems of Geroch's. Indeed, Clarke's uses $\tilde{D}(S)$ in his definition [8, p.119]. Though he uses edgeless hypersurfaces for $S$, this fact does not guarantee that $\tilde{D}(S)$ is open. Krasnikov has also shown that if the free-hole condition is taken as in Geroch's first paper then even Minkowski's spacetime is holed. 9 ]

In a way Clarke's definition is more general, as it does not require any causality condition, not even the acausality of $S$. Nevertheless, a treatment of this case would require 
a considerable generalization of the terminology, together with unnecessary complications, especially whenever $N$ can be chosen totally vicious. For instance, according to the usual definition of horizon, if $N$ is a totally vicious spacetime then $H(\varphi(S))$ is empty although it could be $D(\varphi(S)) \neq N$. Of course, this is not what one usually expect from a domain of dependence, [1] as we use to regard the horizon as the boundary of the Cauchy development.

Clarke [8, Prop. 6.5.1] claims that any inextendible globally hyperbolic spacetime is hole-free. His proof goes as follows. He first considers a globally hyperbolic holed spacetime from which he builds a potentially branched extension of $M$. Using global hyperbolicity he argues that the extension is acceptable as it respects Hausdorffness (thus in the end it is not branched)(this proof is not clear to this author, indeed his set $N^{\prime}$ is closed and the open sets $\pi(U)$ there defined are not homeomorphic to subsets of $\left.\mathbb{R}^{n}\right)$.

We are able to prove the stronger result that inextendible causally simple spacetimes are hole-free. This theorem justifies the intuitive claim that "causal simplicity removes holes from spacetime". Physically it means that any Cauchy hole on spacetime causes some discontinuity in the causal relation.

Theorem II.8. Every inextendible and causally simple spacetime is hole-free.

Proof. Let us suppose, by contradiction, that $(M, g)$ is inextendible, causally simple, and that it has a future hole. Thus there is a partial Cauchy hypersurface $S$, a spacetime $(N, \sigma)$, and an isometry $\varphi: \overline{D(S)} \rightarrow N$ such that $\varphi(S)$ is acausal, and

$$
B:=D^{+}(\varphi(S)) \cap \overline{\varphi\left(H^{+}(S)\right)}=\left[D^{+}(\varphi(S)) \cap \partial \varphi\left(D^{+}(S)\right)\right] \backslash \varphi(S)
$$

is non-empty (Prop. ПI.5).

We remark that starting from any point of $B$ and moving backwards along a timelike curve one enters immediately into $\varphi\left(D^{+}(S)\right)$. Let $r \in D^{+}(\varphi(S)) \cap \varphi\left(H^{+}(S)\right)$ and let $x=\varphi^{-1}(r)$, $x \in H^{+}(S)$. There is a past inextendible geodesic $\eta^{\prime}:(0,1] \rightarrow N$ ending at $r$ such that $\eta:=\left.\varphi^{-1} \circ \eta^{\prime}\right|_{(a, 1]}$ is, for some $a>0$, a past inextendible lightlike generator of $H^{+}(S)$ ending at $x$ (see Fig. 1). Observe that $\eta$ does not intersect $S$, since $S$ is edgeless, nevertheless, $\eta^{\prime}$ intersects $\varphi(S)$ as $r \in D^{+}(\varphi(S))$. Thus following $\eta^{\prime}$ in the backward direction we should reach a last point $p \in B$. Now, let $C$ be a convex neighborhood of $p$ such that $C \subset W \subset$ $D^{+}(\varphi(S)) \backslash \varphi(S)$ where $W$ is causally convex in the globally hyperbolic spacetime $D(\varphi(S))$. Any point $q \in J_{C}^{-}(p) \backslash\{p\}$ cannot belong to $\overline{\varphi\left(H^{+}(S)\right)}$, for this is true for the points staying in $\eta^{\prime} \cap J_{C}^{-}(p)$ and if $q$ is not of this type then it can be connected with a timelike curve $\sigma^{\prime}$ to $r$ (as it can be connected to $r$ through a piecewise geodesic causal curve passing through $p$ with a corner at $p$ ), and hence, if it were $q \in \overline{\varphi\left(H^{+}(S)\right)}$ we could find $q^{\prime} \in \varphi\left(H^{+}(S)\right) \cap C$ and a timelike curve $\beta^{\prime}$ which connects $q^{\prime}$ with $r$. If $\beta^{\prime}$ is entirely in the image of $\varphi$ then the existence of $\beta:=\varphi^{-1} \circ \beta^{\prime}$ contradicts the achronality of $H^{+}(S)$. If instead, $\beta^{\prime}$ is not entirely in the image of $\varphi$ the curve $\varphi^{-1} \circ \beta^{\prime}$ provides a past inextendible causal curve ending at $x$ and not intersecting $S$, again a contradiction. In summary, we have shown that there is a point $p \in B$ and a convex neighborhood $C \ni p, C \subset W \subset D^{+}(\varphi(S)) \backslash \varphi(S)$ such that $\left[J_{C}^{-}(p) \backslash\{p\}\right] \cap \overline{\varphi\left(H^{+}(S)\right)}=\emptyset$ and hence $J_{C}^{-}(p) \backslash\{p\} \subset \varphi\left(D^{+}(S)\right)$. In particular, $p \notin \varphi\left(H^{+}(S)\right)$, for otherwise the image of the generator of $H^{+}(S)$ passing through $\varphi^{-1}(p)$ would provide points belonging to $\left[J_{C}^{-}(p) \backslash\{p\}\right] \cap \varphi\left(H^{+}(S)\right)$.

Let $p_{n} \rightarrow p, p_{n} \in \varphi\left(H^{+}(S)\right)$, and let $\gamma_{n}^{\prime}$ be the past inextendible lightlike geodesic ending at $p_{n}$ such that the pullback $\varphi^{-1} \circ \gamma_{n}^{\prime}$ (with suitably restricted domain) is the generator of $H^{+}(S)$ ending at $\varphi^{-1}\left(p_{n}\right)$. The curves $\gamma_{n}^{\prime}$ converge to a past inextendible curve ending 


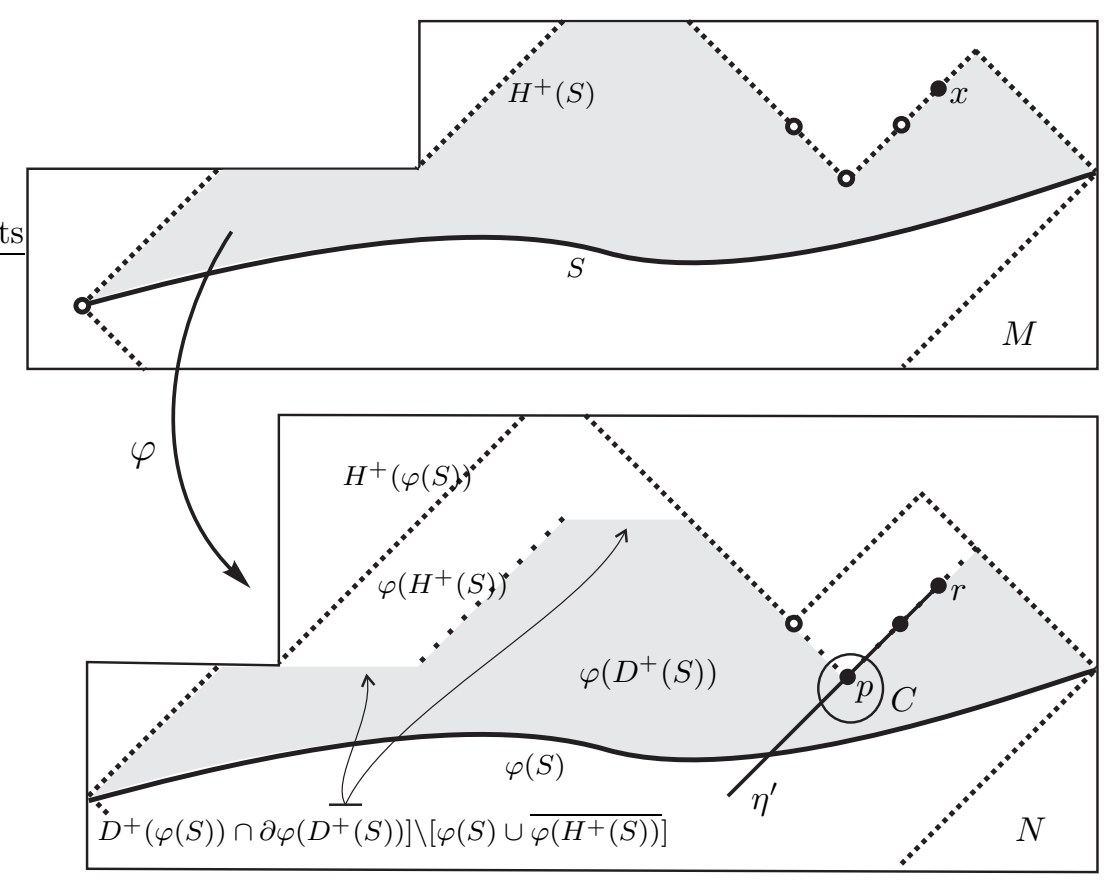

FIG. 1: An example of future holed spacetime obtained by removing some sets from Minkowski $1+1$ spacetime. This example illustrates the definition of hole and the proof of theorem $\llbracket .8$. The circled empty points do not belong to the manifold. If the set indicated by the arrows is non-empty then $M$ can be easily extended.

at $p$. Since there is an open set $V \subset C \cap \varphi\left(D^{+}(S)\right)$ of $J_{C}^{-}(p) \backslash\{p\}$, for sufficiently large $n$ we must have that some $\gamma_{n}^{\prime}$ intersects $V$. Let $N$ be such that there is $b_{N} \in \gamma_{N}^{\prime} \cap V$. Following $\gamma_{N}^{\prime}$ from $p_{N}$ in the past direction we must leave $\overline{\varphi\left(H^{+}(S)\right)}$ before $b_{N}$ at some last point $c$. Point $c$ cannot belong to $\varphi\left(H^{+}(S)\right.$ ) (for otherwise it would not be the last point), thus $c \in \overline{\varphi\left(H^{+}(S)\right)} \backslash \varphi\left(H^{+}(S)\right)$. Then we get a contradiction with causal simplicity because $\left(\varphi^{-1}\left(b_{N}\right), \varphi^{-1}\left(p_{N}\right)\right) \in \overline{J^{+}} \backslash J^{+}$. In order to show that $\left(\varphi^{-1}\left(b_{N}\right), \varphi^{-1}\left(p_{N}\right)\right) \in \overline{J^{+}}$it is sufficient to consider a sequence of points $b_{N}^{k} \rightarrow b_{N}, b_{N}^{k} \ll b_{N}$. We can find timelike curves $\alpha_{k}$ connecting $b_{N}^{k}$ to $p_{N}$, thus entirely contained in the image of $\varphi$. Their pullback shows that $\varphi^{-1}\left(b_{N}^{k}\right) \ll \varphi^{-1}\left(p_{N}\right)$ where $\varphi^{-1}\left(b_{N}^{k}\right) \rightarrow \varphi^{-1}\left(b_{N}\right)$.

Remark II.9. In the previous proof it could be tempting to claim that the pair $\left(\varphi^{-1}(e), x\right)$, where $e$ is the intersection of $\eta^{\prime}$ with $S$, belongs to $\overline{J^{+}} \backslash J^{+}$and provides a pair of points giving the desired contradiction. Unfortunately, this simplified strategy does not work because there could be conjugate points in $\eta^{\prime}$ between $p$ and $r$. Their presence would actually imply $r \in I^{+}(e)$.

Every extendible spacetime is causally geodesically incomplete. If there are holes we have

Theorem II.10. Every inextendible future holed spacetime admits a future lightlike incomplete geodesic and a future timelike incomplete geodesic. These geodesics are contained in $D(S)$ and the Riemann tensor, and its covariant derivatives at any order, evaluated on a parallely transported base over them have a finite limit. 
Proof. We proceed as in the previous proof using the same notation. Any causal future directed geodesic ending at $p$ in $N$ provides, once pulled back to $M$ through $\varphi^{-1}$, the desired incomplete geodesic. Since all these geodesics have image under $\varphi$ which converge to $p$, the Riemann tensor and its covariant derivatives must converge to the value they take on $p$.

\section{A. Examples}

In this section we give some examples which clarify the independence of the concept of hole and inextendibility, and the relative strength of casual continuity, causal simplicity, and hole-freeness.

The dimensionality of the next examples is not really important because one can always increase the dimension with the direct product with $\mathbb{R}^{k}, k \geq 1$, with the Euclidean metric. It is also useful to recall that any non-total imprisoning spacetime can be made causally geodesically complete and hence inextendible through multiplication of the metric by a suitable conformal factor. [10]

Example II.11. An extendible but hole-free spacetime. The region $t<0$ of Minkowski spacetime, or the region $t+x<0$ of Minkowski spacetime.

Example II.12. An inextendible causally continuous spacetime can be holed. Let $M$ be Minkowski $3+1$ spacetime with the origin $o$ removed. The spacetime metric is multiplied by a conformal factor different from unity in $I^{+}(o)$ in such a way that the timelike geodesic $t>0, x=y=z=0$, becomes geodesically complete in the past direction.

Example II.13. Another inextendible but holed spacetime. The covering of $1+1$ Minkowski spacetime minus a point.

Example II.14. An inextendible causally continuous hole-free spacetime need not be causally simple. Plane wave gravitational metrics are known to be causally continuous but not causally simple.[11, 12] Furthermore, they are complete [13, Chap. 13] thus inextendible [13, Prop. 6.16] and by theorem II.10 they are hole-free.

Example II.15. An inextendible hole-free causal spacetime need not be causally continuous. Some generalized gravitational wave metrics are known to be non-distinguishing. [14]

Example II.16. Some holes can be removed extending the spacetime. Minkowski spacetime minus a point.

Example II.17. A future holed spacetime need not be past holed. Minkowski $1+1$ spacetime minus a future directed lightlike ray.

These examples prove that inextendibility and hole-freeness are independent concepts (examples П.11, ПI.12, ПI.13). Moreover, they prove that theorem II.8 is optimal since we cannot weaken the assumption of causal simplicity to causal continuity (example II.12). Finally, they show that the hole-free property cannot find a place in the causal ladder between causal simplicity and causal continuity (example II.15), and that in general holefreeness does not promote causal properties (example \.15).

This fact had to be expected because, while the levels of the causal ladder represent physical conditions on spacetime, the hole-free property is a mathematical requirement which should be placed on the very definition of spacetime and which cannot, by itself, improve causality. Of course, our theorem shows that we could omit to include the hole-free condition 
whenever dealing with globally hyperbolic or causally simple spacetimes. Nevertheless, this condition is expected to be useful for the study of causally continuous and stably causal spacetimes, and in general for spacetimes satisfying weak causality conditions.

\section{CONCLUSIONS}

We have introduced a definition of holed spacetime which removes some undesirable consequences of previous proposals. In particular, we have observed that a hole should generate an horizon $H(S)$ that must in part disappear when we pass to a different spacetime containing $\tilde{D}(S)$. We have then been able to prove that every inextendible causally simple spacetime is hole-free, thus confirming the expectation that causal simplicity removes holes from spacetime. This theorem is optimal as it cannot be improved weakening causal simplicity to causal continuity (example II.12). Physically, this result means that if spacetime contains some partial Cauchy hypersurface which does not completely develop its influence, then there is some discontinuity in the causal relation.

\section{Acknowledgments}

I thank Alfonso García-Parrado Gómez-Lobo and the Centro de Matematica, Universidade do Minho, for kind hospitality. This work has been partially supported by GNFM of INDAM and by FQXi.

[1] S. W. Hawking and G. F. R. Ellis, The Large Scale Structure of Space-Time (Cambridge University Press, Cambridge, 1973).

[2] E. Minguzzi and M. Sánchez, The causal hierarchy of spacetimes, Vol. H. Baum, D. Alekseevsky (eds.), Recent developments in pseudo-Riemannian geometry of ESI Lect. Math. Phys. (Eur. Math. Soc. Publ. House, Zurich, 2008), p. 299, arXiv:gr-qc/0609119.

[3] E. Minguzzi, "Weak distinction and the optimal definition of causal continuity," Class. Quantum Grav. 25, 075015 (2008).

[4] R. Geroch, Prediction in general relativity, Vol. Foundations of Space-Time Theories of Minnesota Studies in the Philosophy of Science, vol. VIII, (University of Minnesota Press, Minneapolis, 1977), p. 81.

[5] J. B. Manchak, "Is spacetime hole-free?" Gen. Relativ. Gravit. 41, 1639 (2009).

[6] C. J. S. Clarke, "Space-time singularities," Commun. Math. Phys. 49, 17 (1976).

[7] J. Earman, Bangs, crunches, whimpers, and shrieks: singularities and acausalities in relativistic spacetimes (Oxford University Press, Oxford, 1995).

[8] C. J. S. Clarke, The analysis of space-time singularities (Cambridge University Press, Cambridge, 1993).

[9] S.V. Krasnikov, "Even Minkowski spacetime is holed," Phys. Rev. D 79, 124041 (2009).

[10] J. K. Beem, "Conformal changes and geodesic completeness," Commun. Math. Phys. 49, 179 (1976).

[11] R. Penrose, "A remarkable property of plane waves in general relativity," Rev. Mod. Phys. 37, 215 (1965). 
[12] P. E. Ehrlich and G. G. Emch, "Gravitational waves and causality," Rev. Math. Phys. 4, 163 (1992).

[13] J. K. Beem, P. E. Ehrlich, and K. L. Easley, Global Lorentzian Geometry (Marcel Dekker Inc., New York, 1996).

[14] V. E. Hubeny, M. Rangamani, and S. F. Ross, "Causally pathological spacetimes are physically relevant," Int. J. Mod. Phys. D14, 2227 (2005). 